\title{
Case report on vulval intraepithelial neoplasia (VIN)
}

\begin{abstract}
This case report concerns a woman diagnosed with Vulval Intraepithelial Neoplasia (VIN) type III. She had been married for 15 years and had conceived for three times, giving birth to three live babies since then. The patient was admitted to HAHC hospital with chief complaints of severe itching in the vulval area since three years and formations of warts in the vulval area since one year. During vulval examination presence of warts was seen and redness was also present around the warts. On histopathological examination it was found that the patient had Vulval Intraepithelial Neoplasia type III (warty type). As a part of management, hemivulvectomy was performed so as to prevent the further worsening of the case. VIN is a condition in which there are particular changes in the skin that covers the vulva. It is also referred to as a premalignant condition. Though VIN is not a cancer but if the changes becomes severe and are left untreated in the patients with VIN, then it might develop into cancer of vulva.
\end{abstract}

Volume 10 Issue 2 - 2019

\author{
Somibala Thokchom, Shivangi Sharma \\ Faculty of Jamia Hamdard, India
}

Correspondence: Shivangi Sharma, MSc Nursing Student, Faculty of Jamia Hamdard,Wz-406-g plot no.7I-a second floor, janak park, hari nagar, New Delhi, India, Tel 8447/91160, Email shivangisharma981 I@gmail.com

Received: February 24, 2017| Published: March 05, 2019

\section{Introduction}

A 37 years old woman from a low socio economic background came to Gynaecology Department of HAHC hospital of New Delhi due to complaints of severe itching over the vulval area since three years and presence of warts with redness around them in the vulval area since one year. She also had complaints of dyspareunia and burning sensation over vulva. She ignored the itching for the first year of its occurrence but then went to a dermatologist and started a treatment for the itching for at least one year, but it was of no use and the itching remained severe. The patient knew little about the treatment and there were no records regarding the medication or treatment with the patient at the time of admission. Her obstetric history included three conceptions with three live births. There was no history of miscarriage. She weighed $54 \mathrm{~kg}$ and has been married for fifteen years. There was no history of consanguineous marriage of parents and there was no family history of any gynaecological disease in her family. Her age at menarche was 14 years and her menstrual cycle was regular. She had no significant past history of diabetes, hypertension or any other medical illness.

On admission the vaginal examination of patient revealed presence of warts with redness around them over the skin covering the vulva. She was admitted on the same day and routine investigations were carried out. All blood test reports were found to be near normal. PAP was also done for the patient to rule out presence of HPV, which also happens to fall under the risk factors and causes of VIN. PAP smear results were found to be negative. Patient was also tested for HIV before being diagnosed with VIN and the result was negative. After all the required clinical tests and proper examination the gynecologists planned for hemivulvectomy and the surgery was performed. In hemivulvectomy the vulval skin along with underlying fat was excised. Vulval skin involving whole of the suspicious area on both right and left side and posteriorly along with anal orifice was excised.

\section{The different degrees of VIN are}

VIN I: Corresponds to mind cellular atypia. The lesion is limited to the deeper one-third of the epithelium.

VIN II:Corresponds to moderate cellular atypia. The lesion is limited up to middle-third of the epithelium.
VIN III: Corresponds to severe cellular atypia and carcinoma-in-situ. The abnormal cells involve whole thickness of the epithelium. There is no stromal invasion. ${ }^{1}$

\section{Incidence}

It is more frequent in patients in age group 20-40 years, i.e. at a younger age group compared to vulval carcinoma. The average age is as low as 33 years. It is often related with STD such as condyloma accuminata, herpes simplex virus II, gonorrhea, syphilis or gardnerella vaginalis.

HPV associated VIN is seen more in young women. HPV 6 and 11 are associated with vulval condylomas. HPV 16, 18, 31, 35 is associated with VIN lesions. There is increased prevalence of associated Cervical Intraepithelial Neoplasia (CIN) (10-80\%).

Regression frequently occurs in young women, during pregnancy or when it is caused by viral infection. Rarely progressive to invasive carcinoma $(<10 \%)$. It takes $20-30$ years to progress to invasive cancer. ${ }^{2}$

\section{ISSVD classification}

The ISSVD gave the classification in 2004 in which only the highgrade disease is classified as VIN.

This is subdivided into:

1) Usual-type VIN (including warty, basaloid and mixed subtypes): Commonly associated with carcinogenic genotypes of HPV and/or HPV persistence factors such as cigarette smoking or immunocompromised states.

2) Differentiated VIN: Commonly associated with vulvar dermatoses such as lichen sclerosis. Differentiated VIN associated with lichen sclerosis, however, is more likely to be associated with squamous carcinoma than is usual-type VIN. ${ }^{3}$

\section{Causes}

The exact cause of VIN is unknown. Studies are being done to determine the cause and recurrence of VIN. The following factors, however, have been associated with $\mathrm{VIN}^{3}$ :

a. Human Papilloma Virus (HPV) 
b. Herpes Simplex Virus Type 2 (HSV-2)

c. Smoking

d. Immunosuppression

e. Chronic Vulvar Irritation

f. Conditions such as Lichen Sclerosus.

\section{Diagnosis}

The chief complaints may be pruritis vulvae, lump or bleeding from a vulval ulcer. It may be even symptomless.

Local examination reveals a lesion in the vulva with white, grey, pink or dull red colour. Lesions look rough, raised from the surface and often multifocal. Application of 5 percent acetic acid turns VIN lesions white with punctuation and mosaic patterns. These changes are best seen with a colposcope.

Cytologic screening of the vulva is not useful and unreliable. Confirmation of diagnosis is done by biopsy. Usually 3-5 mm diameter

\begin{tabular}{l} 
S. No. Book picture \\
\hline $\begin{array}{l}\text { A persistent itch in the vulva is the most common type. } \\
\text { The itch may become severe if left untreated. }\end{array}$ \\
$3 \quad$ Soreness, burning or tingling sensation in the vulva. \\
$4 \quad$ Changed appearance of the affected parts of the vulva. \\
5 \\
Redness or white areas of the skin of the vulva.
\end{tabular}

\section{Treatment}

The fact remains that VIN has got low malignant potential. The risk of progression to invasive disease is less than 10 percent. Hence conservative management is generally accepted.

\section{Medical}

Medical treatment is done for young woman to control the symptoms. Topical fluorinated steroid ointment can be applied twice daily for a period of about 6 months. Commonly used preparations are 0.1 percent triamcinolone acetonide, or 0.1 percent betamethasone valerate. Local application of chemotherapy in the form of 5 percent fluorouracil (5-FU) cream may be used in recurrent lesions. The result is however unsatisfactory.

Whatever therapy is employed, it is mandatory for regular follow up. Biopsies to be taken freely whenever an abnormal area is detected. ${ }^{2,4}$

\section{Surgery}

Following types of surgeries can be done:

A. Local excision: Wide local excision with $1 \mathrm{~cm}$ margin is reserved in young patient with localized lesion.

B. Laser Therapy: $\mathrm{CO}_{2}$ laser vaporization is gaining increasing popularity in the treatment of vulval epithelial atypia. It gives better cosmetic results with lower recurrence rate. In multifocal dermal punch is taken under local anesthetic. The small amount of bleeding i controlled using Monsel's solution (ferrous sulphate). Larger biopsy when required may be taken using a scalpel. Multiple site biopsies are useful.

A complete pelvic examination is to be done. To exclude vaginal or cervical neoplasia, cytologic evaluation has to be performed. ${ }^{2}$

\section{Histology}

The cells exhibit features of malignancy. There is complete loss of polarity and stratification. Cellular immaturity, nuclear abnormalities and mitotic activity vary depending upon the grade of VIN. There is hyperkeratosis, acanthosis (hyperplasia of epidermis) and chronic inflammatory cell infiltration. Koilocytes may be present. The rete ridges are large and elongated. Basement membrane remains intact. There is no evidence of involvement of the dermis. ${ }^{2}$

\section{Symptoms}

Sometimes there are no obvious symptoms, particularly when it first develops. However, symptoms eventually do develops. ${ }^{4}$

\section{Patient picture}

It was present in the patient since 3 years.

Soreness and burning sensation over vulval skin was present in the patient.

Present in patient.

Formation of itchy warts and lesions over the patient's vulva changed the appearance of her vulval skin.

Redness around the warts over vulva of the patient was present.

lesion, simple excision of the individual lesion is done. Skinning vulvectomy is less commonly done. Cryosurgery and loop electrosurgical excision procedure (LEEP) has been used.

C. Simple vulvectomy is employed in diffuse type especially in postmenopausal women. Long term follow up is needed as the risk of recurrence is high (40-70\%).

D. Ablation therapy is alternatives to surgery. Ablation means destroying the affected area. It can be done using high energy beam (laser) or a tiny electrical current passed through a probe.

E. Photodynamic therapy is another treatment which is occasionally used. For this treatment, a medication is either applied tropically to the vulva or given as an injection. The medication is taken up by the abnormal cells and is light sensitive. A few hours later, a cold laser light is shown at the abnormal cells. This activates the light sensitive medication, which has an effect of destroying the abnormal cells.

F. It is also best to avoid using soaps, deodorants etc., on the vulval skin as these can be irritating. To wash the vulva a bland moisturizer such as emulsifying ointment can be used instead of soap.

\section{Prevention}

Vaccinating young girls with HPV vaccine before their initial sexual contact has been claimed to reduce the incidence of VIN. ${ }^{3}$ 


\section{Prognosis of VIN}

All the treatments have a good chance of clearing vulval intraepithelial neoplasia (VIN). Even with successful treatment, there is a fair chance that VIN may return at some point in the future. This is why regular follow ups after the treatments are very important.

\section{Acknowledgments}

None.

\section{Conflicts of interest}

The authors declare no conflicts of interest.

\section{References}

1. Preti M, Scurry J, Marchitelli CE, et al. Vulval Intraepithelial Neoplasia. Best Pract Res Clin Obstet Gynaecol. 2014;28(7):1051-1062.

2. Dutta DC. Textbook of Gynaecology. In: Hiralal Konar, editor. Jaypee Brothers Medical Publishers; 2014:318-320.

3. https://en.wikipedia.org/wiki/Vulvar_intraepithelial_neoplasia

4. Van Seters M, Van Beurden M, Ten Kate FJ, et al. Treatment of Vulval intraepithelial neoplasia with topical imiquimod. $N$ Engl $J$ Med. 2008;358(14):1565-1473. 